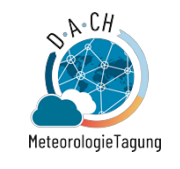

Kurzfassungen der Meteorologentagung DACH

DACH2022-149, 2022

$\mathrm{DACH} 2022$

(C) Author(s) 2021. This work is distributed under

the Creative Commons Attribution 4.0 License.

\title{
Impact of Tropical Convective conditions on Solar Irradiance Forecasting based on Cloud Motion Vectors
}

Arindam Roy, Annette Hammer, Detlev Heinemann, Ontje Lünsdorf, and Jorge Lezaca German Aerospace Center (DLR), Institute of Networked Energy Systems, Carl-von-Ossietzky-Str., 15, 26129 Oldenburg, Germany

Cloud Motion Vector ( $\mathrm{CMV}$ ) estimation from consecutive satellite images is widely used commercially for providing hours-ahead intraday forecasts of solar irradiance and PV power production. The modelling assumptions in these methods are generally satisfied for advective motion which is common in mid-latitudes, but strained for tropical meteorological conditions dominated by convective clouds. The region under analysis in this study encompasses both tropical and subtropical climatic zones and is affected by seasonal strong convection, i.e., the South Asian Monsoon.

The purpose of this study is to benchmark the monthly forecast error of three commonly used CMV estimation techniques - Block-match, Farnebäck (Optical flow) and TV-L ${ }^{1}$ (Optical flow), for analysing their performance on a seasonal basis. The main focus of this work is the analysis of the limitations of image processing based Block-match and Optical flow techniques in predicting irradiance during the Monsoon period, which presents frequent convective formation and dissipation.

Forecasted Cloud Index (CI) maps are validated against reference analysis CI maps for the period 2018-2019 for forecast lead times from 0 to 5.5 hours ahead using the Peak Signal to Noise Ratio (PSNR) metric for estimating the accuracy. Persistence of analysis cloud index maps are used as the reference worst case scenario forecast. Site-level forecasts of irradiance for the same period are validated against ground measured irradiance from two BSRN stations - Gurgaon and Tiruvallur, located in Northern and Southern India respectively.

From the Winter period in March to the Monsoon period in August, there is a marked reduction of the 30 minutes ahead forecast accuracy by $3 \mathrm{~dB}$ in terms of Peak Signal to Noise Ratio at the imagewide level. This can be observed for all the three methods and the worst-case persistence scenario. Both optical flow methods outperform Block-match by $0.5 \mathrm{~dB}$ for the entire period of analysis. The Gurgaon BSRN site is affected by Summer Monsoon and shows an increase in nRMSE by a factor of 3 for all the methods. This station shows a seasonal pattern of forecast error closely matching the image-wide forecast accuracy. The forecast error for the Tiruvallur BSRN station on the other hand reaches its peak in December (Data for October and November are absent), due to its location in the Winter Monsoon climatic zone. Again, the nRMSE for all methods increase by a factor of almost 3 from March to December. The inter-method difference in accuracy is not significant and a seasonal difference $(20 \%$ nRMSE) dominates. This highlights the shortcomings of image processing techniques in extrapolating future cloud locations under convective situations, where there is rapid change in cloud content between consecutive images. 